\title{
Satisfiability and Integer Programming As Complementary Tools *
}

\author{
Ruiming Li, Dian Zhou \\ Department of Electrical Engineering \\ School of Engineering and Computer Sciences \\ The University of Texas at Dallas \\ Richardson, TX 75080 \\ $\{$ rl18, zhoud $\} @$ utdallas.edu
}

\author{
Donglei Du \\ Faculty of Administration \\ University of New Brunswick \\ P. O. Box 4400 \\ Fridericton, NB E3B 5A3 \\ New Brunswick, Canada \\ ddu@unb.ca
}

\begin{abstract}
Satisfiability (SAT) and integer linear programming (ILP) are two related NP-complete problems. They both have a lot of important applications. We study the effectiveness of using them as a complementary tool to each other. We propose three different ILP formulations to solve SAT and compare them with state-ofthe-art SAT solvers Berkmin and zchaff. On the other hand, we give two methods to solve ILP by using SAT solvers. In both cases, we achieve speed-ups of several orders for most of our tested examples.
\end{abstract}

\section{INTRODUCTION}

Integer linear programming (ILP) and Satisfiability (SAT) are among the most important NP-complete problems. SAT, the first problem shown to be NP-complete [10], becomes a hot research topic in the EDA community [7, 8], and several efficient SAT solvers are developed [5, 22, 9, 19, 17, 23]. The most successful SAT solvers such as Berkmin [5] and zchaff [23] can solve real application problems with tens of hundreds variables in a reasonable time. These solvers are based on the basic Davis Putnam Logemann Loveland (DPLL) backtracking procedure [11]. On the other hand, there is no algorithm that can efficiently solve every problem which is NP-complete. A heuristic algorithm that is efficient for these problems may not be good for the other problems. In this paper, we study the effectiveness of using SAT and ILP as a complementary tool to each other.

SAT problem can be easily transformed to ILP. For example, $\left(x_{1}+\overline{x_{2}}+x_{3}\right)\left(\overline{x_{1}}+\overline{x_{3}}+x_{5}\right)\left(x_{2}+x_{4}+x_{5}\right)$ can be written as the following inequality constraints,

$$
\begin{cases}x_{1}-x_{2}+x_{3} & \geq 0 \\ -x_{1}-x_{3}+x_{5} & \geq-1 \\ x_{2}+x_{4}+x_{5} & \geq 1\end{cases}
$$

This provides us an alternative tool for solving SAT by using ILP. On the other hand, given an ILP problem, we can also transform it to a SAT problem in polynomial time by the NPcomplete theory [10]. In [21], the author gave a linear-time transformation algorithm from linear inequalities to conjunctive normal form (CNF). As we know, the worst case running time for NP-complete problems is exponential unless $\mathrm{P}=\mathrm{NP}$. This means we can not expect any SAT and ILP solvers to

\footnotetext{
${ }^{*}$ This research is supported by NSF CCR-0098275, CCR-0306298.
}

solve any SAT and ILP problems, respectively, in reasonable time. Because of the close relationship between SAT and ILP, it may be beneficial and effective to apply the methods available for one problem to solve the other. Our results in this paper strongly support this idea.

This paper is organized as follows. In section 2 and 3, we will discuss how to use ILP to solve SAT, and how to use two SAT-based methods to solve ILP, respectively. We conclude our paper in section 4 .

\section{ILP APPLIED TO SAT}

We can transform a SAT problem to an equivalent ILP in many different ways, like the one we did in (1.1). The transformed ILP then can be solved by using any ILP solver, such as CPLEX [16], which is widely used in the public domain. We introduce three transformations based on different objective functions as follows.

Formulation I: minimize 0 , subject to $A X \geq 1$.

Formulation II: minimize $x_{0}$, subject to $x_{0}+A X \geq 1$.

The original SAT is satisfiable iff the min value of $x_{0}$ is 0 .

Formulation III: minimize $0 X$, subject to $A X \geq 1$, where $0 X=0 x_{1}+0 x_{2}+\cdots+0 x_{n}$.

We use some benchmarks [15] to show the performance of these three different formulations with state-of-the-art SAT solvers Berkmin561 and zchaff, which are among the best SAT solvers in 2003 and 2002 SAT competitions. In this paper, all the experiments are implemented on SUN Ultra 10 Workstation, OS 5.8 with $128 \mathrm{M}$ of RAM memory, and 440MH CPU. Version 8.1.0. of CPLEX is used. The results are shown in Table I and II. The column CPLEX Formulation I in table I is the running time using CPLEX for the above formulation I. The columns CPX I, II, and III in table II are the running time using CPLEX for the formulation I, II, and III, respectively. From Table I, we can see CPLEX outperforms Berkmin and zchaff for these FPGA routing instances. Table II shows that the performance can be different for different formulation I, II and III.

\section{SAT APPLIED TO ILP}

There are two ways to solve ILP by SAT solver. One is to transform ILP to SAT directly. After the transform, any efficient SAT solver can be applied to solve the transformed SAT

(C) 2004 IEEE. Personal use of this material is permitted. However, permission to reprint/republish this material for advertising or promotional purposes or for creating new collective works for resale or redistribution to servers or lists, or to reuse any copyrighted component of this work in other works must be obtained from the IEEE. 
TABLE I

RESULTS FOR ILP FORMULATION I AND COMPARISON WITH BERKMIN AND ZCHAFF

\begin{tabular}{|c|c|l|c|c|c|}
\hline $\begin{array}{c}\text { benchmark } \\
\text { name }\end{array}$ & $($ Var, Cls) & $\begin{array}{c}\text { SAT or } \\
\text { UNSAT }\end{array}$ & $\begin{array}{c}\text { CPLEX } \\
\text { Formulation I(sec) }\end{array}$ & $\begin{array}{c}\text { zchaff } \\
\text { time(sec) }\end{array}$ & $\begin{array}{c}\text { BerkMin561 } \\
\text { time(sec) }\end{array}$ \\
\hline fpga10_11_uns_rcr & $(220,1122)$ & UNSAT & 0.04 & 1385.56 & 97.32 \\
\hline fpga10_12_uns_rcr & $(240,1344)$ & UNSAT & 0.05 & 1532.72 & $>50000$ \\
\hline fpga10_13_uns_rcr & $(260,1585)$ & UNSAT & 0.05 & $>50000$ & $>50000$ \\
\hline fpga10_15_uns_rcr & $(300,2130)$ & UNSAT & 0.07 & $>50000$ & $>50000$ \\
\hline fpga10_20_uns_rcr & $(400,3840)$ & UNSAT & 0.11 & $>50000$ & $>50000$ \\
\hline fpga11_12_uns_rcr & $(264,1476)$ & UNSAT & 0.06 & $>50000$ & $>50000$ \\
\hline fpga11_13_uns_rcr & $(286,1742)$ & UNSAT & 0.07 & $>50000$ & $>50000$ \\
\hline fpga11_14_uns_rcr & $(308,2030)$ & UNSAT & 0.07 & $>50000$ & $>50000$ \\
\hline fpga11_15_uns_rcr & $(330,2340)$ & UNSAT & 0.07 & $>50000$ & $>50000$ \\
\hline fpga11_20_uns_rcr & $(440,4220)$ & UNSAT & 0.14 & $>50000$ & $>50000$ \\
\hline
\end{tabular}

TABLE II

COMPARISON RESULTS FOR DIFFERENT ILP FORMULATIONS

\begin{tabular}{|c|c|c|c|c|}
\hline $\begin{array}{c}\text { benchmark } \\
\text { name }\end{array}$ & $\begin{array}{c}\text { SAT or } \\
\text { UNSAT }\end{array}$ & $\begin{array}{c}\text { CPX I } \\
(\mathrm{sec})\end{array}$ & $\begin{array}{c}\text { CPX II } \\
(\mathrm{sec})\end{array}$ & $\begin{array}{c}\text { CPX III } \\
(\mathrm{sec})\end{array}$ \\
\hline aim-100-1_6-no-1 & UNSAT & 36.34 & 1.32 & 91.80 \\
\hline aim-100-1_6-no-2 & UNSAT & 0.50 & 1.23 & 36.05 \\
\hline aim-100-1_6-no-3 & UNSAT & 8.20 & 1.51 & 15.23 \\
\hline aim-100-1_6-no-4 & UNSAT & 1.79 & 0.28 & 4.26 \\
\hline aim2001_6-no-1 & UNS & 10.15 & 0.79 & $>2000$ \\
\hline aim2001_6-no-2 & UNS & 1.19 & 3.01 & 956.46 \\
\hline fpga10_11_uns_rcr & UNS & 0.04 & $>1000$ & 0.04 \\
\hline fpga10_11_uns_rcr & UNS & 0.05 & $>1000$ & 0.05 \\
\hline fpga12_8_sat_rcr & SAT & 0.13 & 0.22 & 0.09 \\
\hline fpga12_9_sat_rcr & SAT & 0.10 & 0.25 & 0.20 \\
\hline fpga12_10_sat_rcr & SAT & 0.19 & 0.30 & 0.15 \\
\hline fpga13_10_sat_rcr & SAT & 0.07 & 1.00 & 0.20 \\
\hline
\end{tabular}

problem. This method considers the SAT solver as a blackbox and its efficiency depends on the performance of transformation algorithm and the SAT solver being used. Any improvement to SAT solver can benefit ILP solution. In [21], a linear time transformation algorithm from linear inequalities to CNF was given. We will extend this result to solve general 0-1 ILP.

Another method is to embed the SAT-based advanced branch-and-bound search techniques into ILP searching procedures. We use a real-life application problem to show its efficiency. Several papers discussed how to use the SAT-based search techniques to solve an 0-1 ILP [1] [2] [4] [3][6].

\section{A. Transforming ILP to SAT}

Warners [21] proposed an algorithm to transform the following linear inequality to CNF,

$$
\sum_{i=1}^{m} a_{i} x_{i} \leq b,
$$

where $x_{i} \in\{0,1\}, a_{i}(1 \leq i \leq m)$ is a positive natural number such that $\max _{1 \leq i \leq m} \bar{a}_{i}=2^{N}$, and $b$ is an integer. They first transform the left hand side $\sum_{i=1}^{m} a_{i} x_{i}$ into CNF. Then with
TABLE III

TRANSFORMING ILP TO SAT VS.CPLEX

\begin{tabular}{|c|c|c|c|}
\hline $\begin{array}{c}\text { Benchmark } \\
\text { Name }\end{array}$ & $\begin{array}{c}\text { (Vars, } \\
\text { Rows) }\end{array}$ & $\begin{array}{c}\text { CPLEX } \\
\text { time(sec) }\end{array}$ & $\begin{array}{c}\text { zchaff } \\
\text { time(sec) }\end{array}$ \\
\hline acc-tight:4 & $(1620,3286)$ & $>50000$ & 256.37 \\
\hline acc-tight:5 & $(1339,3053)$ & 2090.15 & 620.24 \\
\hline acc-tight:6 & $(1335,3048)$ & 949.93 & 399.81 \\
\hline markshare1 & $(62,7)$ & $>50000$ & 12.31 \\
\hline markshare2 & $(74,8)$ & $>50000$ & 95.48 \\
\hline
\end{tabular}

this result, transform $\leq b$ into CNF. To do the first step, just recursively call the procedure that transforms

$$
a_{1} x_{1}+a_{2} x_{2},
$$

into CNF.

Although the efficiency to solve ILP by using SAT was demonstrated by the Frequency Assignment Problem (FAP) in [21], the reason why we restate it here is because the SAT solvers have made a great progress in recent years. We try to see how ILP can be benefited by using the state-of-the-art SAT solvers. In [4], a similar result to transform ILP to SAT was also proposed, but the experimental result by using SAT as a blackbox to solve transformed CNF was not shown.

After transforming all the constraints in ILP to CNF, the binary search method is used for the objective function whose lower and upper bound can be obtained by linear programming relaxation. To compare the performance, SAT solver zchaff with the newest version as of July 1, 2003 and state-of-the-art ILP commercial solver CPLEX with version 8.1.0 are used. The experimental results are shown in Table III for the problems in [13] and the ACC family in [14]. From Table III, we can see that SAT method is very efficient for the tested benchmarks for which CPLEX can not solve within a very large time period. Theoretically, we can extend this method to solve mixed integer programming (MIP) by representing each nonbinary variable with binary variables, and following the similar procedure.

Warners' transformation algorithm from (3.1) to CNF needs to introduce a lot of additional variables. As further considerations, more efficient transformation algorithm from ILP to 
$\mathrm{CNF}$ that results in fewer number of variables and clauses may exist. But Hook [12] already proved that it is a NP-complete problem if no addition variable is introduced. Hence if the coefficients or the number of terms in the constraints are very large, this method might be inefficient. In the next section, we study how to apply SAT-based search techniques to ILP.

\section{B. Apply SAT-Based Search Techniques To ILP}

As we know, the branch-and-bound search method is also a basic method for solving ILP. The advancement of SAT may result in improvements for ILP. We compare the branch-andbound method for SAT with that for ILP. The decision making heuristic for both ILP and SAT should be similar, i.e. a decision strategy that is good for SAT is most likely good for ILP also. We can apply the most effective decision making strategy of SAT to ILP. During the search procedure for ILP, a conflict is encountered if the value of left-hand side (LHS) is larger than that of right-hand side (RHS) for the type of " $\leq$ " constraints or the value of LHS is less than that of RHS for the type of " $\geq$ " constraints. For objective function, we consider it as a constraint for its known upper or lower bound. The similar strategies for BCP, conflict analysis, and learning in SAT can be applied to ILP.

In the next, we apply the SAT-based search techniques to the following real-life application example extracted from sensor networks [20]. This will demonstrate how to effectively use the advanced SAT search techniques to solve ILP, by making full use of the characteristics of the given problem.

Maximum Set Cover Partition Problem. Given a set $A=$ $\{1, \cdots, n\}$ of $n$ elements to be covered and a collection $\mathcal{C}=$ $\left\{C_{1} \cdots, C_{m}\right\}$ of subsets of $A$, a cover of $A$ is a collection of sets such that all elements of $A$ are included in their union. The objective is to partition $\mathcal{C}$ into maximum number of covers for A.

This problem is NP-complete as shown in [20]. We use a small example to illustrate this problem. Suppose $A=$ $\{1,2,3,4,5,6\}$, and $\mathcal{C}=\left\{C_{1}, C_{2}, C_{3}, C_{4}, C_{5}\right\}$, where $C_{1}=$ $\{1,2,4,6\}, C_{2}=\{1,2,3,4,5\}$, and $C_{3}=\{1,3,5,6\}, C_{4}=$ $\{2,3,4,6\}, C_{5}=\{1,2,3,4,5,6\}$. There are several partitions for $\mathcal{C}$ each of which is a cover of $A$. For instance, both $\left\{C_{1}, C_{2}\right\}$ and $\left\{C_{3}, C_{4}, C_{5}\right\}$ are partitions of $C$, each of which covers $A$, but they are not optimal. The optimal solution is 3 that partitions $C$ into 3 sets: $\left\{C_{1}, C_{2}\right\},\left\{C_{3}, C_{4}\right\}$, and $\left\{C_{5}\right\}$.

We formulate the maximum set cover partition problem as an integer linear program as follows.

Let us fix the number of partition as $K$, and denote $G_{1}, \cdots, G_{K}$ to be the $K$ disjoint covers of $A . n=|A|$, and $m=|\mathcal{C}|$, where $|$.$| is the cardinality.$

Let

$$
x_{j k}= \begin{cases}1, & \text { if } C_{j} \in G_{k}, j=1, \cdots, m, k=1, \cdots, K ; \\ 0, & \text { otherwise. }\end{cases}
$$

Since any set $C_{j}$ in $\mathcal{C}$ belongs to at most one $G_{k}$, we have,

$$
\sum_{k=1}^{K} x_{j k} \leq 1, j=1, \cdots, m .
$$

and each element $i$ in $A$ must be covered by at least one set in every group $G_{k}, k=1, \cdots, K$, then

$$
\sum_{j: i \in C_{j}} x_{j k} \geq 1, \quad i=1, \cdots, n, k=1, \cdots, K
$$

Hence the ILP is the following:

Maximize $K$

Subject to

$$
\begin{cases}\sum_{k=1}^{K} x_{j k} \leq 1, & j=1, \cdots, m \\ \sum_{j: i \in C_{j}} x_{j k} \geq 1, \quad i=1, \cdots, n, & k=1, \cdots, K \\ x_{j k} \in\{0,1\} . & \end{cases}
$$

To solve this ILP problem, we can use CPLEX. But it is not efficient, generally, if the number of variables is very large.

We propose a branch and bound search (BBS) method based on the satisfiability search techniques. We solve this ILP by binary search for the value of $K$. Each time, we solve a feasible problem. Let $\operatorname{deg}(i)$ be the number of sets which contain $i$, then an obvious upper of $K$ is $\underset{i=1, \cdots, n}{\min } \operatorname{deg}(i)$.

In [18], SAT-based search technique based on GRASP solver [19] was successfully applied to the binary covering problem. Now we adapt the zchaff search strategies to solve the K-covering problem that is different from general set covering problems in that the constraints of (3.3) is different from clauses in SAT. In order to apply the SAT search method, we need to compare the constraints in (3.3) and (3.4) with those in SAT. We can easily see that each constraint in (3.4) is equivalent to a SAT clause. Instead of directly transforming the constraints in (3.3) into CNF as we did in the previous section, we try to deal with them within the branch and bound search procedure. We find that the constraints in (3.3) are more powerful in propagating variables compared to boolean constraints. In fact, if one of them is assigned to 1 , then all other variables in the same constraint must be assigned to 0 immediately. For example, suppose we have a constraint, $x_{1}+x_{3}+x_{4}+x_{6}+x_{7} \leq 1$, if there is an assignment $x_{3}=1$ during the search, then we must have $x_{1}=0, x_{4}=0, x_{6}=0$ and $x_{7}=0$. This greatly speeds up the algorithm.

We implement the algorithm by utilizing the same decision making, $\mathrm{BCP}$ and conflict learning strategies as those in the zchaff solver. The difference is the treatment of the constraints in (3.3) as we just discussed in the above. For comparing purpose, we also run the PBS program [4] which also studied the SAT-based search method to ILP. The test examples are generated randomly.

The experimental result is shown in table IV. The column SAT-BBS is our SAT-based branch and bound search method. From Table IV, we can see that our SAT-based method is extremely successful, which is better than both CPLEX and PBS. It is faster in several orders than CPLEX, and the reason that our program is better than PBS is that our algorithm is more application-specific. 
TABLE IV

SAT-BASED BRANCH AND BOUND VS.CPLEX FOR ILP

\begin{tabular}{|c|c|c|c|c|c|}
\hline$|C|$ & $|A|$ & $\begin{array}{c}\text { (vars, } \\
\text { rows })\end{array}$ & $\begin{array}{c}\text { CPLEX } \\
(\mathrm{sec})\end{array}$ & $\begin{array}{c}\text { SAT-BBS } \\
(\mathrm{sec})\end{array}$ & $\begin{array}{c}\text { PBS } \\
(\mathrm{se})\end{array}$ \\
\hline 40 & 2000 & $(600,2040)$ & 21.60 & 0.01 & 0.1 \\
\hline 60 & 2100 & $(900,2160)$ & 91.94 & 0.01 & 0.1 \\
\hline 80 & 3360 & $(1200,3440)$ & 215.98 & 0.01 & 0.1 \\
\hline 100 & 4000 & $(1500,4100)$ & 427.57 & 0.02 & 0.1 \\
\hline 120 & 4800 & $(1800,4920)$ & 635.78 & 0.02 & 0.1 \\
\hline 140 & 6300 & $(2100,6440)$ & 1243.38 & 0.02 & 0.12 \\
\hline 160 & 7200 & $(2400,7360)$ & 2010.47 & 0.03 & 0.12 \\
\hline 180 & 9000 & $(3600,9180)$ & MEM OUT & 0.04 & 0.14 \\
\hline 200 & 11000 & $(4000,1240)$ & MEM OUT & 0.05 & 0.14 \\
\hline
\end{tabular}

\section{CONCLUSION}

We have demonstrated that ILP can be an effective tool for solving SAT as shown in section 2. In fact, there is a rich theory for ILP in the operation research fields. But its application to SAT is still very limited thus far. Our future research will focus on this area. On the other hand, SAT can also be an effective tool for ILP. Our result of section 3 shows that it is very important to design algorithms that make full use of the special properties of the given problems. We can conclude that, for ILP and SAT, any progress made in one problem can improve the other correspondingly.

\section{REFERENCES}

[1] P. Barth, A Davis-Putnam based Enumeration Algorithm for Linear Pseudo-Boolean Optimization, Technical Report MPI-I-95-2-003, Max-PlanckInstitut Fr Informatik, 1995.

[2] P. Barth, OPBDP: A Davis-Putnam based Enumeration Algorithm for Linear Pseudo-Boolean Optimization, http://www.mpi-sb.mpg.de/units/ag2/software lopbdp.

[3] H. Xu, R. Rutenbar, K. Sakallah, sub-SAT: A Formulation for Relaxed Boolean Satisfiability with Applications in Routing, in Proc. of the Int'l Symposium on Physical Design, 2002.

[4] F. Aloul, A. Ramani, I. Markov, and K. Sakallah, Generic ILP versus Specialized 0-1 ILP: an Update, ICCAD 2002.

[5] E. Goldberg, and . Novikov, BerkMin: A Fast and Robust Sat-Solver, 2002 Design, Automation and Test in Europe Conference and Exhibition (DATE'02)

[6] D. Chai and A. Kuehlmann, A fast pseudo-boolean constraint solver, Proc. of the Design Automation Conference, pp. 830-835, 2003.

[7] S. Devadas, Optimal layout via boolean satisfiability, IEEE/ACM ICCAD, pp 294-297, 1989.
[8] R.Wood and R. A. Rutenbar, FPGA routing and routability estimation via boolean satisfiability. IEEE Tran. VLSI, pp. 222-231, June, 1998.

[9] R. Bayardo, and R. Schrag, Using CSP lookback techniques to solve real-world SAT instances, Proc. of the 14th Na.(US) Conf. on Artificial Intelligence(AAAI-97), AAAI Press/The MIT Press, 1997.

[10] S.A.Cook, The complexity if theorem proving procedures, Proceedings of 3rd Annual ACM Symposium on the Theory of Computing, New York, 1971,pp:151-158.

[11] M. Davis, G. Logemann, and D. Loveland, A Machine Program for Theorem Proving, Communications of the ACM, 5(7), pp. 394-397, 1962.

[12] J. N. Hook, unpublished note.

[13] http://www.caam.rice.edu/ bixby/miplib/ miplib3.html

[14] http://www.ps.uni-sb.de/ walser/acc/acc.html

[15] http://www.eecs.umich.edu/ faloul/ benchmarks.html

[16] ILOG CPLEX, http://www.ilog.com/products/cplex

[17] M. Moskewicz, C. Madigan, Y. Zhao, L. Zhang, and S. Malik, Chaff: Engineering an Efficient SAT Solver, in Proc. of the Design Automation Conference, pp. 530-535, 2001.

[18] V. Manquinho, J. Marques-Silva, Search pruning techniques in SAT-based branch-and-bound algorithms for the binate covering problem, IEEE Transactions on Computer-Aided Design of Integrated Circuits and Systems, Vol. 21(5), pp. 505 -516, May 2002.

[19] J.P. Marques-Silva and K.A. Sakallah, GRASP: A Search Algorithm for Propositional Satisfiability, IEEE Tranctions on Computers, vol.48,506-521, 1999.

[20] S. Slijepcevic and M. Potkonjak, Power efficient organization of wireless sensor networks. IEEE International Conference on Communications, Vol.2,pp.472-476, 2001.

[21] Joost P. Warners, A linear-time transformation of linear inequlities into conjunctive normal form. Information Processing Letters 68(1998)63-69.

[22] H.Zhang, SATO: an efficient proposition solver, Proceedings of the International Conference on Automated deduction, July 1997.

[23] L.Zhang, C.Madigan, M.Moskewicz and S.Malik, Efficient conflict driven learning in a Boolean satisfiability solver. IEEE/ACM International Conference on Computer Aided Design, pp.279 -285, 2001. 